\title{
Relación entre el intervalo de nacimiento entre gemelos y el pH de cordón umbilical: ¿existe asociación entre la acidosis del primer gemelo y el segundo?
}

\author{
Víctor Lago Leal ${ }^{1}$, Vanesa Ocaña Martínez ${ }^{1}$, Luis Martínez Cortés ${ }^{1}$, Miguel Ángel \\ Huertas Fernández. ${ }^{1}$ \\ 1 Servicio de Obstetricia, Servicio de Obstetricia y Ginecología, Hospital Universitario de Getafe, España.
}

\section{RESUMEN}

Objetivos: Determinar si el intervalo de nacimiento vía vaginal entre gemelos, se relaciona con un descenso de $\mathrm{pH}$ arterial/venoso de cordón umbilical del segundo gemelo respecto al primero, y establecer si el pH acidótico del primer gemelo predice la acidosis del segundo. Método: Estudio de cohortes retrospectivo en 77 pares de gemelos realizado en el Hospital de Getafe, España, en el periodo 2008-2013. Se relacionó el tiempo transcurrido entre el nacimiento de los gemelos con el resultado del pH de cordón y el exceso de bases. Estudiamos la correlación entre el tiempo transcurrido entre ambos nacimientos y la diferencia entre los valores de $\mathrm{pH}$ del primer y segundo gemelo, y la correlación con la diferencia de exceso de base. Resultados: Hubo correlación lineal significativa entre el tiempo transcurrido entre el nacimiento de los gemelos y la diferencia de $\mathrm{pH}$ venoso del segundo gemelo respecto del primero $(R=0,560 ; p=0,0001)$, así como con el $\mathrm{pH}$ arterial $(\mathrm{R}=0,502 ; \mathrm{p}=0,0001)$. El $\mathrm{pH}<7,20$ del primer gemelo se asoció con acidosis en el cordón del $2^{\circ}$ gemelo $(<7,20, p=0,000 ;<7,15, p=0,0001 ;<7,10, p=0,0001 ;<7,05, p=0,001 ;<7,00, p=0,0001)$. Conclusiones: El pH del segundo gemelo siempre fue igual o menor que el del primer gemelo, hubo una correlación lineal entre el intervalo de nacimiento entre gemelos y el descenso del $\mathrm{pH}$ venoso y arterial del segundo gemelo respecto al primero. El pH acidótico del primer gemelo predice la acidosis del segundo.

\section{PALABRAS CLAVE: Intervalo de tiempo en parto gemelar, acidosis fetal, pH de cordón umbilical, estado ácido-base fetal}

\section{SUMMARY}

Objectives: Determinate if the twin-to-twin vaginal delivery time interval, is related with a decline of the arterio-venous cord blood $\mathrm{pH}$ of the second twin regarding to the first twin, and to evaluate if the acidotic $\mathrm{pH}$ of first twin can predict the acidosis status of the second one. Method: A retrospective descriptive cohort study was performed at the Hospital of Getafe, Spain, in the period 2008-2013, with 77 pairs of twins that were registered and met the inclusion criteria. The twin-to-twin delivery time interval and the cord blood status results were registered. A statistical analysis was performed to study the relation of the twin-to-twin delivery time interval with the $\mathrm{pH}$ value differences between first and second twins, and with the base excess. Results: A positive linear correlation between the twin-to-twin delivery time interval and the difference of venous $\mathrm{pH}$ of the second twin respect to the first one was found $(\mathrm{R}=0.560 ; \mathrm{p}=0.0001)$, and also with the difference on arterial $\mathrm{pH}(\mathrm{R}=0.502 ; \mathrm{p}=0.0001)$. A pH value $<7.20$ of the first twin was associated with blood cord acidosis of the second twin $(<7.20, p=0.000 ;<7.15, p=0.0001 ;<7.10, p=0.0001 ;<7.05, p=0,001$; $<7.00, \mathrm{p}=0.0001)$. Conclusions: The second twin $\mathrm{pH}$ was equal to or less than the first twin $\mathrm{pH}$ in all cases. There is a linear relation between twin-to-twin delivery time interval and the decrease of the $\mathrm{pH}$ value of the second twin regarding to the first one. The acidosis of first twin could predict the acidosis status of the second twin.

KEY WORDS: Twin-to-twin delivery time interval, fetal acidosis, umbilical cord $\mathrm{pH}$, fetal acid-base status 


\section{INTRODUCCIÓN}

Alrededor del $10 \%$ de las complicaciones perinatales suceden en partos gemelares, pese a que la prevalencia de este tipo de gestación oscila entre el $1-2,5 \%(1,2)$. La mortalidad es de 3 a 6 veces mayor respecto a gestaciones únicas, en particular el segundo gemelo tiene mayor riesgo de un resultado perinatal adverso (3-6).

Existen diversas teorías para justificar la susceptibilidad a la hipoxia del segundo nacido. Tras el nacimiento del primer gemelo una reducción brusca del volumen uterino puede causar una contracción miometrial que disminuya la superficie de vascularización placentaria o un desprendimiento parcial de placenta por disminución de la superficie de contacto útero-placentaria $(7,8)$. Eliminando el factor de prematuridad el $75 \%$ de las muertes fetales a término en segundos gemelos son debidas a hipoxia intraparto tras el nacimiento del primer gemelo. Siendo el riego absoluto para muerte perinatal del segundo gemelo de 1/270 (1/350 para muerte por hipoxia intraparto, y de $1 / 500$ por hipoxia intraparto debido a distocia), existiendo un riesgo mayor de muerte por asfixia en segundos gemelos nacidos por vía vaginal respecto a cesárea $(4,9)$.

El intervalo de nacimiento entre gemelos ha sido sugerido como factor de riesgo importante asociado con la incidencia de complicaciones perinatales del segundo nacido (10). En nuestra práctica clínica diaria observamos que el pH del cordón del segundo gemelo siempre es igual o menor que el del primero, sobre este hecho postulamos que si el primer gemelo padece un estado acidótico, el estado ácido-base del segundo será igual o más acidótico.

El objetivo de este estudio es determinar si la acidosis del primer gemelo se relaciona con la del segundo y su capacidad de predicción, así como establecer si el intervalo de tiempo entre nacimientos afecta el equilibrio ácido-base entre ambos gemelos.

\section{PACIENTES Y MÉTODO}

Seleccionamos todos los partos gemelares en un periodo de 6 años, recogiendo los datos de la base informatizada del Hospital Universitario de Getafe, España, el cual asistió a una media de 2164 nacimientos al año en el periodo de estudio (2008-2013), con los siguientes criterios de exclusión: 1. Nacimiento del primer gemelo mediante cesárea. 2. Contraindicación de parto vaginal. 3. Muerte anteparto de alguno de los gemelos. 4. Crecimiento intrauterino retardado, transfusión fetofetal o malformación fetal mayor de cualquiera de los dos gemelos.
Se recabaron datos epidemiológicos como edad materna, paridad, peso de los recién nacidos (RN), complicaciones materno-fetales, edad gestacional, y las relativas al parto como corionicidad, maniobras obstétricas, analgesia, presentación, registro cardiotocográfico, duración de expulsivo y tipo de parto.

La toma de sangre arterial y venosa fetal, se realizó mediante doble pinzamiento precoz del cordón, inmediatamente antes de iniciada la actividad respiratoria. Para medir la determinación de $\mathrm{pH}$ de cordón se utilizó un equipo EM Premier 4000 (IL, Instrumentation Laboratory).

Se tomó el tiempo transcurrido entre el nacimiento de los gemelos, y se recogió el resultado de $\mathrm{pH}$ de cordón y exceso de bases. Estudiamos la correlación entre el tiempo transcurrido entre ambos nacimientos (intervalo entre gemelos) y la diferencia de $\mathrm{pH}$ (medida como diferencia entre los valores del primer y segundo gemelo). También estudiamos la correlación con la diferencia de excesos de base (medida también como diferencia entre los valores de ambos gemelos).

El análisis estadístico se realizó mediante el programa SPSS Version 19.0 (IBM Corp., Armonk NY). Se utilizaron las pruebas de $T$ de Student y $U$ de Mann-Whitney para analizar las características de ambos grupos. Se calculó la relación entre la diferencia del $\mathrm{pH}$ del segundo gemelo respecto al primero y el intervalo de nacimiento entre ambos, mediante análisis de correlación de Pearson y el test exacto de Fisher para relacionar los $\mathrm{pH}$ de los primeros y segundos gemelos. El estudio fue aprobado por el Comité de Ética e Investigación Clínica de la institución.

\section{RESULTADOS}

Fueron seleccionados 77 casos. La media de la edad materna fue $32,6 \pm 4,1$ años, la edad gestacional media fue de $35,89 \pm 2,07$ semanas. El $77,92 \%$ fueron gestaciones bicoriales-biamnióticas, y el $22,08 \%$ a gestaciones monocoriales-biamnióticas.

El $84,4 \%$ de las pacientes se les administró analgesia epidural. Se realizó episiotomía a las pacientes siguiendo criterios restrictivos en el $55,4 \%$ de la muestra. La presentación del segundo gemelo fue cefálica en 59 casos $(76,5 \%)$, podálica en 13 casos $(16,9 \%)$ y 5 casos en transversa/oblicua $(6,5 \%)$.

En la Tabla I se presentan las características del parto de la población de estudio. No hubo diferencias entre el peso al nacimiento de los gemelos. Teniendo en cuenta el inicio de la fase de expulsivo desde la dilatación completa del primer gemelo, la duración media de la fase de expulsivo de los primeros gemelos fue de $63,31 \pm 59,1$ min y para los segundos gemelos de 78,19 $\pm 62,93 \mathrm{~min}$. 
Tabla I

CARACTERÍSTICAS DEL PARTO EN 77 PARES DE GEMELOS

\begin{tabular}{|c|c|c|c|}
\hline \multirow{3}{*}{$\begin{array}{l}\text { Variables } \\
\text { Peso al nacimiento: }\end{array}$} & \multicolumn{3}{|c|}{ Características del parto } \\
\hline & Primer gemelo (n: 77) & Segundo gemelo (n: 77) & Valor $p$ \\
\hline & $2340,46 \pm 414,64 \mathrm{~g}$ & $2300,78 \pm 439,25 \mathrm{~g}$ & $\left(^{*}\right) p=0,0567$ \\
\hline \multirow[t]{2}{*}{ Registro cardiotocográfico: } & Primer gemelo (n: 75) & Segundo gemelo (n: 76) & \\
\hline & Tranquilizador: 68 (90,6\%) & Tranquilizador: 64 (84,2\%) & \\
\hline \multirow[t]{3}{*}{ 1h antes del expulsivo } & No tranquilizador: $7(9,4 \%)$ & No tranquilizador: $12(15,8 \%)$ & \\
\hline & Patológico: 0 (0\%) & Patológico: $0(0 \%)$ & \\
\hline & Tranquilizador: 68 (90,6\%) & Tranquilizador: 44 (57,9\%) & \\
\hline \multirow[t]{2}{*}{ Expulsivo } & No tranquilizador: $7(9,4 \%)$ & No tranquilizador: $27(35,5 \%)$ & \\
\hline & Patológico: $0(0 \%)$ & Patológico: 5 (6,6\%) & \\
\hline \multirow[t]{2}{*}{ Duración del expulsivo: } & Primer gemelo (n: 77) & Segundo gemelo (n: 77) & \\
\hline & $63,31 \pm 59,1 \mathrm{~min}$ & $78,19 \pm 62,93 \mathrm{~min}$ & $\left(^{* \star}\right) p=0,027$ \\
\hline Tipo de parto: & Primer gemelo (n: 77) & Segundo gemelo (n: 77) & \\
\hline Eutócico & $57(74 \%)$ & $53(68,8 \%)$ & \\
\hline Instrumental & $20(26 \%)$ & $7\left(9,1^{\circ} \%\right)$ & \\
\hline Podálica & & $10(13 \%)$ & \\
\hline Cesárea & & $7(9,1 \%)$ & \\
\hline
\end{tabular}

(*) T de Student. (**) U de Mann-Whitney

Hubo diferencias estadísticamente significativas en la media del $\mathrm{pH}$ arterial y del $\mathrm{pH}$ venoso de los primeros y segundos gemelos, siendo menor la de este último en ambos casos $(p=0,0001)$. No hubo diferencias entre las medias de exceso de bases (EB) (Tabla II). En nuestra serie encontramos que en todos los casos, el pH tanto arterial como venoso siempre fueron igual o más bajos en el segundo gemelo.

La media de intervalo de nacimiento entre gemelos fue de $16,08 \pm 12,45$ min (rango: 2-75 minu- tos). Respecto a la diferencia entre los $\mathrm{pH}$ de ambos gemelos, el $\mathrm{pH}$ venoso ( $\mathrm{n}$ : 75/77) del segundo gemelo fue de media $0,089 \pm 0,08416$ más bajo que el del primero, así como el pH arterial ( $\mathrm{n}: 52 / 77$ ) que fue de media $0,0879 \pm 0,09042$ más bajo. En relación a la diferencia entre el exceso de base (EB) de ambos gemelos, el EB venoso (n: 64/77) y arterial ( $\mathrm{n}: 44 / 77)$ del segundo gemelo fue de media $0,6750 \pm 3,036 \mathrm{mEq} / \mathrm{L}$ y $1,4455 \pm 3,38 \mathrm{mEq} / \mathrm{L}$ más bajo que el del primero, respectivamente.

Tabla II

VALORES ANALÍTICOS DEL CORDÓN UMBILICAL DEL PRIMER Y SEGUNDO GEMELAR

\begin{tabular}{cccc}
\hline ARTERIAL & Primer gemelo $( \pm 2 \mathrm{DS})$ & Segundo gemelo $( \pm 2 \mathrm{DS})$ & Valor $p$ \\
\hline $\mathrm{pH}$ & $7,2743 \pm 0,134$ & $7,1837 \pm 0,23$ & $\left(^{\star}\right) p=0,0001$ \\
$\mathrm{~EB}(\mathrm{mEq} / \mathrm{L})$ & $-5,2622 \pm 6,12$ & $-8,2473 \pm 25,99$ & $\left(^{\star *}\right) p=0,073$ \\
\hline VENOSO & Primer gemelo $( \pm 2 \mathrm{DS})$ & Segundo gemelo $( \pm 2 \mathrm{DS})$ & Valor $p$ \\
\hline $\mathrm{pH}$ & $7,3236 \pm 0,12$ & $7,2345 \pm 0,23$ & $\left(^{\star}\right) p=0,0001$ \\
EB $(\mathrm{mEq} / \mathrm{L})$ & $-6,1875 \pm 5,53$ & $-8,2028 \pm 23,31$ & $\left(^{\star *}\right) p=0,279$ \\
\hline
\end{tabular}

(*) U de Mann-Whitney. $\left({ }^{\star \star}\right) \mathrm{T}$ deStudent. 
La correlación entre el tiempo transcurrido entre el intervalo de los nacimientos con la diferencia de $\mathrm{pH}$ venoso entre los gemelos fue directa y significativa $(R=0,560 ; p<0,0001)$ (Figura $1 A)$, similar observación se aprecia en el intervalo entre los nacimientos con la diferencia de $\mathrm{pH}$ arterial $(\mathrm{R}=0,502$; $p=0,0001$ ) (Figura 1C). La diferencia de exceso de bases venoso se correlaciona directa y significativamente con el intervalo entre los nacimientos $(R=0,341 ; p=0,023)$ (Figura 1B). No hubo correlación entre el intervalo de los nacimientos con la diferencia de exceso de base arterial entre ambos nacimientos $(R=0,234 ; p<0,063)$ (Figura 1D).

Estudiamos la relación entre la acidosis del cordón de sangre arterial y venosa del primer gemelo $(\mathrm{pH}<7,20)$ y la acidosis de cordón del segundo gemelo a distintos puntos de corte $(\mathrm{pH}: 7,20 ; 7,15$; $7,10 ; 7,05 ; 7,00)$. Encontramos una relación estadísticamente significativa entre la acidosis de cordón del primer gemelo $(\mathrm{pH}<7,20)$ y la acidosis del cordón umbilical del segundo nacido para todos los puntos de corte estudiados (prueba exacta de Fisher: 7,20; $p=0,0001 ;<7,15 ; p=0,0001 ;<7,10 ; p=0,0001 ;<7,05$; $p=0,001 ;<7,00 ; p=0,0001)$.

\section{DISCUSIÓN}

Nuestros resultados evidencian que la condición ácido-base del segundo gemelo experimenta un deterioro directamente proporcional al tiempo transcurrido desde el nacimiento del primer gemelo. Encontramos la existencia de una correlación lineal significativa entre el tiempo transcurrido entre el nacimiento de los gemelos y la diferencia de $\mathrm{pH}$ venoso y arterial del segundo gemelo respecto del primero, siendo ambas correlaciones de fuerza moderada ( $R=0,560$ y 0,502 respectivamente). Así mismo encontramos una correlación lineal significativa débil $(R=0,341)$ entre el tiempo transcurrido entre el nacimiento de los gemelos y el deterioro de exceso de bases venoso del segundo gemelo respecto del primero.

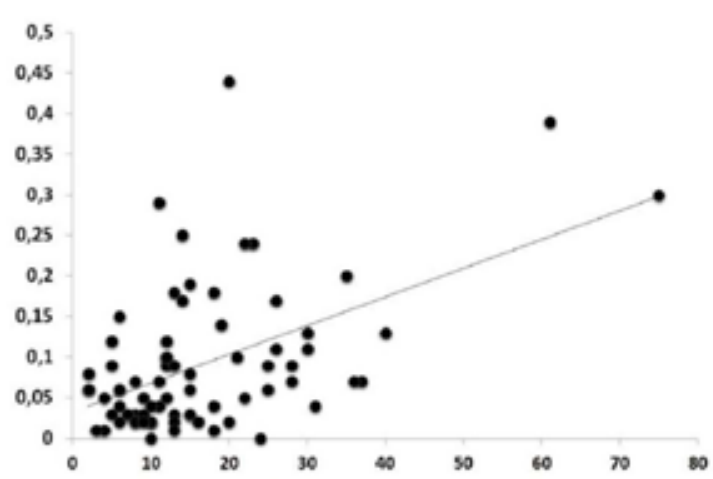

A) Correlación entre la diferencia de $\mathrm{pH}$ venoso (eje $\mathrm{Y}$ ) vs. intervalo de nacimiento entre gemelos (eje $\mathrm{X}$ )

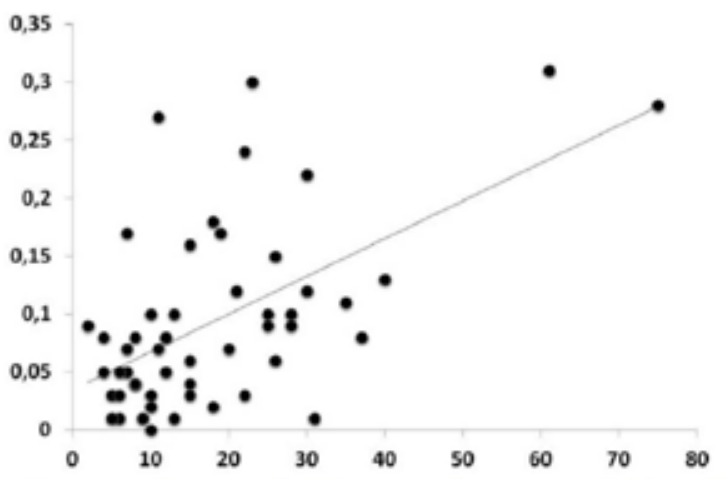

C) Correlación entre la diferencia de pH arterial (eje $Y$ ) vs. intervalo de nacimiento entre gemelos (eje $\mathrm{X}$ )

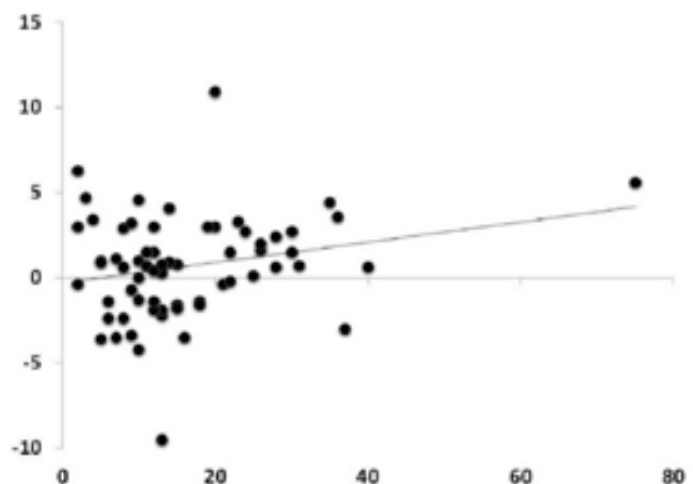

B) Correlación entre la diferencia de EB venoso (eje $Y$ ) vs. intervalo de nacimiento entre gemelos (eje $X$ )

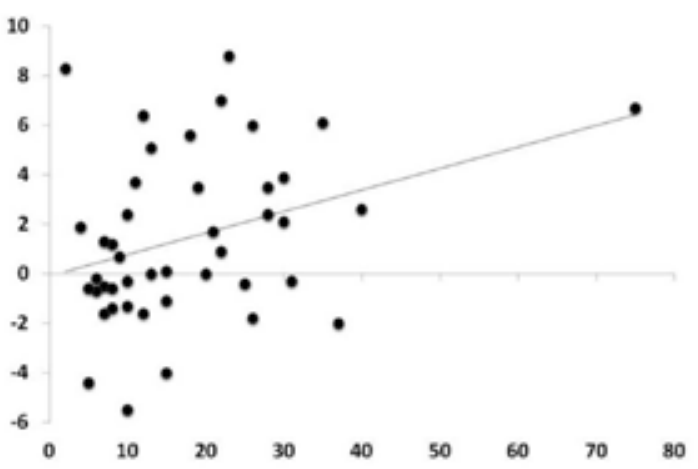

D) Correlación entre la diferencia de EB arterial (eje $\mathrm{Y}$ ) vs. intervalo de nacimiento entre gemelos (eje X)

Figura 1. Correlación lineal del intervalo de nacimiento entre gemelos con las diferencias de los datos analíticos de la sangre de cordón umbilical entre gemelos. 
En la cohorte de estudio el $\mathrm{pH}<7,20$ del primer gemelo se asoció con acidosis en sangre de cordón del cordón $2^{\circ}$ gemelo, estos datos fueron obtenidos mediante el test exacto de Fisher, por tanto sus conclusiones deben ser interpretadas con cautela. Siendo necesarios estudios con una casuística mayor en la serie que permita un análisis estadístico más potente.

Respecto a estudios previamente publicados con un objeto similar de estudio, algunos autores concluyen que el $\mathrm{pH}$ del segundo gemelo es más bajo que el del primero $(1,3,7,11-16)$ y parece deteriorarse con más rapidez (17), también describen una correlación negativa entre el intervalo de nacimiento entre gemelos y las diferencias en el $\mathrm{pH}$ de ambos gemelos o el valor del pH de cordón del segundo (11,17-20). Sin embargo, no tienen en consideración el estado ácido-base del primer gemelo, ni el deterioro del $\mathrm{pH}$ de cordón umbilical del segundo gemelo respecto al primero. Según nuestra hipótesis, el equilibrio ácido base del segundo gemelo está relacionado con el del primero y se deteriora conforme aumenta el tiempo de nacimiento entre ambos; así transcurrido el mismo tiempo entre el nacimiento de dos pares de gemelos, no es lo mismo que partamos de un equilibrio ácido base normal del primer gemelo, a que partamos de un equilibrio ácido base alterado, pues en este último caso el segundo gemelo tendrá un $\mathrm{pH}$ más patológico, con las consecuencias que esa situación pudiere conllevar para el segundo nacido.

\section{CONCLUSIÓN}

Nuestros resultados señalan que existe una correlación directa y significativa entre el tiempo transcurrido entre el nacimiento de los gemelos y la disminución del pH de cordón del segundo gemelo. El estado ácido-base del segundo gemelo siempre es más acidótico que el del primero. El análisis del $\mathrm{pH}$ de cordón inmediatamente tras el nacimiento del primer gemelo podría ser útil en el manejo del parto del segundo gemelar.

\section{REFERENCIAS}

1. De Veciana M, Major C, Morgan MA. Labor and delivery management of the multiple gestation. Obstet Gynecol Clin North Am 1995;22:235-46.

2. Boggess KA, Chisholm CA. Delivery of the non vertex second twin: a review of the literature. Obstet Gynecol Surv 1997;52:728-35.

3. Ferguson WF. Perinatal mortality in multiple gestations: a review of perinatal deaths from 1609 multiple gestations. Obstet Gynecol 1964;23:861-70.
4. Smith GC, Fleming KM, White IR. Birth order of twins and risk of perinatal death related to delivery in England, Northern Ireland, and Wales, 1994-2003: retrospective cohort study. BMJ 2007;334(7593):576.

5. Ware HH 3rd. The second twin.Am J Obstet Gynecol1971;110:865-73.

6. Wen SW, Fung Kee Fung K, Oppenheimer L, et al. Neonatal morbidity in second twin according to gestational age at birth and mode of delivery. Am J Obstet Gynecol 2004;191:773-7.

7. Cunningham FG, MacDonald PC, Gant NF, Leveno KJ, Gilstrap LC, Hankins GD, Clark SL (eds): Williams Obstetrics. 20th ed. Norwalk (CT), Appleton \& Lange, 1997;745-82

8. Maymon R, Herman A, Halperin R, Bukovsky I, Weinraub Z, Ariely S. Changes in uterine artery Doppler flow velocity waveforms during the third stage of labor. Gynecol Obstet Invest 1995;40:24-7.

9. Smith GC, Pell JP, Dobbie R. Birth order, gestational age, and risk of delivery related perinatal death in twins: retrospective cohort study. BMJ 2002;325(7371):1004.

10. Stein W, Misselwitz B, Schmidt S. Twin-to-twin delivery time interval: influencing factors and effect on short-term outcome of the second twin. Acta Obstet Gynecol Scand 2008;87:346-53.

11. Crespo Esteras R, Martínez Suñer S, LaprestaMoros M, Cruz Guerreiro E, Garrido Fernandez P, Campillos Maza JM. Repercusión en los resultados neonatales del intervalo de tiempo de nacimiento entre gemelos. Prog Obstet Ginecol 2011;54:446-451

12. Brown HL, Miller JM Jr, Neumann DE, Sarpong DF, Gabert HA. Umbilical cord blood gas assessment of twins. Obstet Gynecol 1990;75:826-9.

13. Young BK, Suidan J, Antoine C, Silverman F, Lustig I, Wasserman J. Differences in twins: the importance of birth order. Am J ObstetGynecol 1985;151:915-23.

14. Nakano R, Takemura $\mathrm{H}$. Birth order in delivery of twins. Gynecol Obstet Invest 1988;25:217-22.

15. Brown HL, Miller JM, Neumann DE, Sarpong DF, Gabert HA. Umbilical cord blood gas assessment of twins. Obstet Gynecol 1990;75:826-9.

16. Young BK, Duidan J, Antonine C, Silverman F, Lustig I, Wasserman J. Differences in twins: the importance of birth order. Am J Obstet Gynecol 1985;151:915-21.

17. Leung TY, Lok IH, Tam WH, Leung TN, Lau TK. Deterioration in cord blood gas status during the second stage of labour is more rapid in the second twin than in the first twin. BJOG 2004;111:546-9.

18. Suh YH, Park KH, Hong JS, Yoon BH, Shim SS, Park $\mathrm{JS}$, et al. Relationship between twin-to-twin delivery interval and umbilical artery acid-base status in the second twin. J Korean Med Sci 2007;22:248-53.

19. Leung TY, Tam WH, Leung TN, Lok IH, Lau TK. Effect of twin-to-twin delivery interval on umbilical cord blood gas in the second twins. BJOG 2002;109:63-7.

20. McGrail CD, Bryant DR. Intertwin time interval: How it affects the immediate neonatal outcome of the second twin. Am J Obstet Gynecol 2005;192:1420-2. 